Journal of Engineering and Applied Sciences 14 (13): 4617-4624, 2019

ISSN: 1816-949X

(C) Medwell Journals, 2019

\title{
Novel Approach to Solve the Inverse Kinematics Problem for a Multi-Degree-of-Freedom Robotic Arm
}

\author{
Firas S. Hameed, Hassan M. Alwan and Qasim A. Ateia \\ Department of Mechanical Engineering, University of Technology, Baghdad, Iraq
}

\begin{abstract}
In robotics, it is well known that we cannot design a fully operative robotic system without having a complete idea about its kinematics, especially, the inverse kinematics, considering the fact that a successful task accomplishment of the robotic arm is completely dependent upon the accuracy of the end effector positioning along the pre-specified path at every time step. In this study, a new method is proposed to solve the inverse kinematics problem for nth-degree-of-freedom robotic arms. The basic idea of the method is to select a polynomial whose constants are dependent upon the available work space in which the robotic arm operates and align the joints of the robotic arm on that spatial curve. The method showed a perfect performance for robotic arms with many degrees of freedom. However, a five degree of freedom robotic arm was used here to demonstrate the performance of the method.
\end{abstract}

Key words: Inverse kinematics, robotic arm, accuracy, degrees of freedom, accomplishment, performance

\section{INTRODUCTION}

Inverse kinematics of robots studies the spatial configurations of the robotic system that enable its end effector to reach some desired point within the working space of the robot. For a two DOF robotic arm, the inverse kinematics problem is easy to solve because there are only two possible configurations to move the end effector to the point of interest, elbow-up and down. For robotic arms with more degrees of freedom, the inverse kinematics problem becomes much more complicated and finding a solution to the problem becomes rather a difficult task. That's why the inverse kinematics problem must be taken into account in the design stage of any robotic arm. Many approaches have been adopted to solve the inverse kinematics problem. For example, Crenganis et al. (2014) Drexler (2016), Raj et al. (2015) and Das and Mahapatra (2015) used fuzzy logic technique to determine an acceptable solution to the IKP (Ligutan et al., 2017). As it is known about fuzzy logic, so, many rules must be used for each joint in order to find a solution that is nearest to the required one. However, the end effector position obtained may not be guaranteed to follow exactly the desired trajectory points.

Artificial neural networks were also used to solve the inverse kinematics problem (Duka, 2014; Csiszar et al., 2017; Raj et al., 2015; Proceedings of the 2017 Congress on Chinese Automation (CAC), 2017). Those researchers made minor modifications on the structure of the ANNs they used to enhance their performance. However, the classical drawbacks of using the ANNs, although, reduced but could not be avoided completely. For an
ANN to work well, the training set used for the learning process must be large enough in number. This makes the convergence to a solution slow in general. Despite huge in number, the training set is usually only a subset of the total points in the working space of the robotic arm which can make the output of the ANN unpredictable when the end effector is required to pass on points not included in the training set and the ANN needs to be retrained. Also, using the ANNs does not eliminate the possibility of having a multiple solutions for the same end effector point which requires an intervention or a decision to be made by the operator to filter out the repeated solutions of the same end effector position from the training set.

Denavit-Hartenberg's kinematic representation is another approach used by many researchers. Patil et al. (2017), Das and Mahapatra (2015), Basu and Padage (2017), Wang and Wang (2017) tried using the geometrical approach by using Denavit-Hartenberg's kinematic representation of a 5DOF robotic arm. However, they couldn't avoid the problem of solving the set of nonlinear equations with multiple possible solutions. In fact, Iliukhin et al. represented the kinematics of the robotic arm by fixing two joint angles and the inverse kinematic solution they presented is reduced to the simple elbowup-elbow-down one.

Numerical methods are also popular for solving the inverse kinematics problem. These approaches where used by Reiter et al. (2018) and Drexler (2016), for example. Since, numerical methods make use of the highly nonlinear kinematic equations, they are usually not guaranteed to yield an acceptable solution because for

Corresponding Author: Firas S. Hameed, Department of Mechanical Engineering, University of Technology, Baghdad, Iraq 
each point on the path, the iteration scheme is repeated but the resulting joint vector values for one point can be highly different from the joint vector values for the next point on the trajectory which makes the overall motion highly unstable. As the number of trajectory points increases, the time for obtaining a solution for the inverse kinematics problem for the total motion increases as well because the iterations can take a long time to converge.

From the above discussion, it is clear that, although, fuzzy logic, artificial neural networks and numerical methods are the most commonly used methods to obtain an acceptable solution to the IKP each of these methods has its benefits as well as drawbacks.

In this study, we use a novel approach to find a solution to the inverse kinematics problem for an nth DOF robotic arm. For this type of robots, the aforementioned methods usually take a very long time to get the suitable IKP solution, if they could find any. The proposed method enables the robotic arm to accomplish its tasks and move the end effector to the desired position quickly and accurately.

Consider the robotic arm shown in Fig. 1 which has four links with lengths $\mathrm{L}_{1}-\mathrm{L}_{4}$. The whole assembly has the ability to rotate around the base (Joint 0 ) through $360^{\circ}$. It is required to drive the robot in a way, so that, the end effector reaches the desired point $\mathrm{E}=\left(\mathrm{E}_{\mathrm{z}}, \mathrm{E}_{\mathrm{y}}, \mathrm{E}\right)_{\mathrm{z}}^{\mathrm{T}}$. Assuming a planar motion of the robotic is in the $\mathrm{Y}-\mathrm{Z}$ plane which is free from any obstacles, a second order polynomial is used. It has the form:

$$
z(y)=a_{0}+a_{1} y+a_{2} y^{2}
$$

This form is proposed to describe the configuration of the robotic arm joints. There are infinite second order polynomials described by Eq. 1 that can be used depending on the values assigned to the coefficients

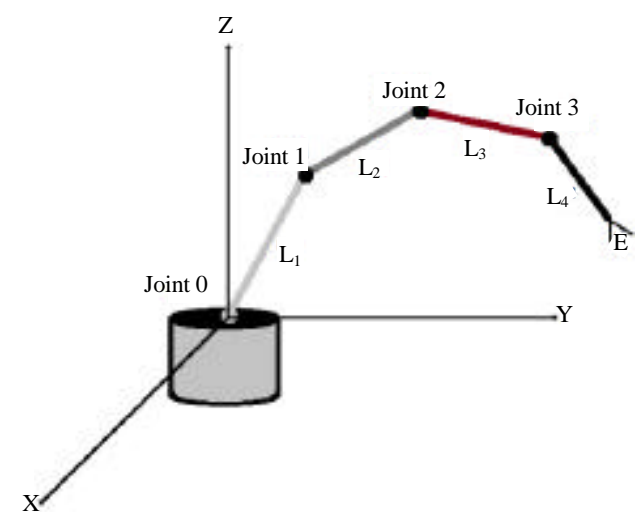

Fig. 1: 5 DOF robotic arm assembly $a_{0}-a_{2}$. However, there is only one combination of those coefficients that guarantees the two important constraints which are:

- The polynomial must connect the base point $(0,0,0)$ and the desired end effector point $\mathrm{E}=\left(0, \mathrm{E}_{\mathrm{y}}, \mathrm{E}_{\mathrm{z}}\right)^{\mathrm{T}}$

- All the four links with their lengths $\mathrm{L}_{1}, \ldots, \mathrm{L}_{4}$ should fit on the polynomial starting from the base $(0,0,0)$ and ending at the end effector desired point $\mathrm{E}$

\section{MATERIALS AND METHODS}

Description of the method: Considering the two constraints mentioned in the previous study, the values of the polynomial coefficient will be determined as follows:

Since, the polynomial passes through the origin $(0,0,0), a_{0}=0$. For the other two coefficients to be evaluated, two equations are needed. One of these equations is readily available, since, the polynomial passes through $\mathrm{E}=\left(0, \mathrm{E}_{\mathrm{y}}, \mathrm{E}_{\mathrm{z}}\right)^{\mathrm{T}}$ which gives:

$$
\mathrm{E}_{\mathrm{z}}=\mathrm{a}_{1} \mathrm{E}_{\mathrm{y}}+\mathrm{a}_{2} \mathrm{E}_{\mathrm{y}}^{2}
$$

To get another equation we need to locate another point on the curve. Let's assume that the coordinates of point (D) are known, that is we assign point (D) the trial coordinate set $\left(0, D_{y}, D_{z}\right)$. As shown in Fig. 2, a trial location for (D) can be found by varying the angle $(\theta)$ within the interval $0-360^{\circ}$. Substituting $\left(D_{y}\right)$ and $\left(D_{z}\right)$ in Eq. 1 a second (but it still a trial) equation will be used to determine a trial values of $a_{1}$ and $a_{2}$ of the polynomial expressed in Eq. 1. The trial coordinates of point $\mathrm{D}$ are given by:

$$
\begin{aligned}
& \mathrm{D}_{\mathrm{y}}=\mathrm{E}_{\mathrm{y}}+\mathrm{L}_{4} \operatorname{Cos}(\theta) \\
& \mathrm{D}_{\mathrm{z}}=\mathrm{E}_{\mathrm{z}}+\mathrm{L}_{4} \operatorname{Sin}(\theta)
\end{aligned}
$$

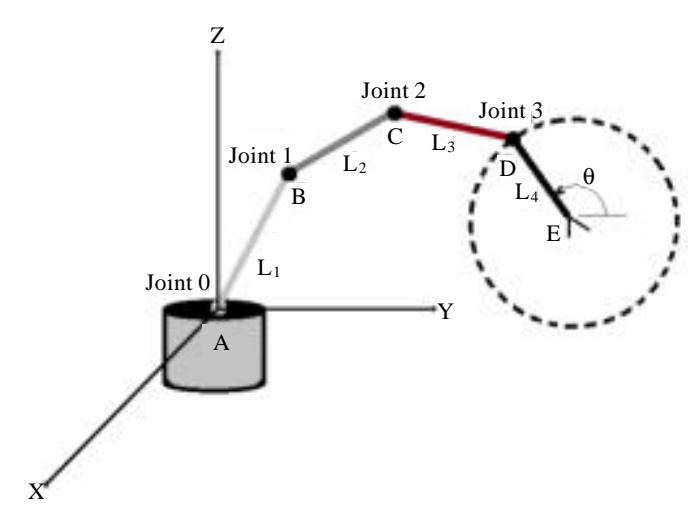

Fig. 2: Locating point D 


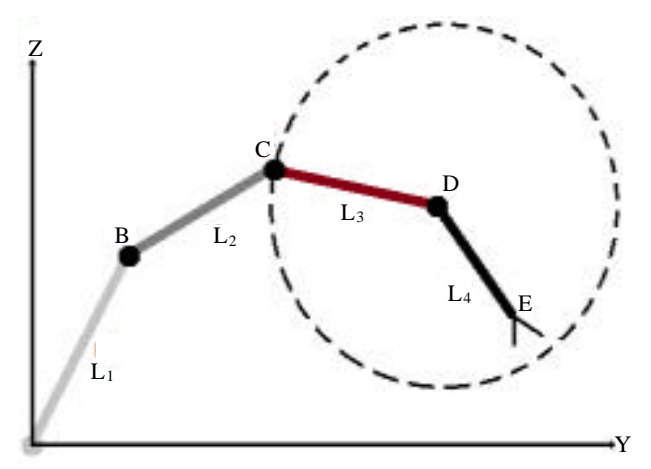

Fig. 3: Intersection of the circle (Eq. 8) with the parabola (Eq. 1)

Since, the assumed point D belongs to Eq. (1), this gives us:

$$
D_{z}=a_{1} D_{y}+a_{2} D_{y}^{2}
$$

Note that the Eq. 3 and 4 are in fact, the parametric equations that represent a circle with radius $\left(\mathrm{L}_{4}\right)$ centered at the end effector position $\mathrm{E}=\left(0, \mathrm{E}_{\mathrm{y}}, \mathrm{E}_{\mathrm{z}}\right)^{\mathrm{T}}$. Equation 2 and 5 can be solved together to determine the first trial coefficients $a_{1}$ and $a_{2}$. Expressing, Eq. 2 and 5 in matrix form gives:

$$
\left[\begin{array}{cc}
\mathrm{E}_{\mathrm{y}} & \mathrm{E}_{\mathrm{y}}^{2} \\
\mathrm{D}_{\mathrm{y}} & \mathrm{D}_{\mathrm{y}}^{2}
\end{array}\right]\left[\begin{array}{l}
\mathrm{a}_{1} \\
\mathrm{a}_{2}
\end{array}\right]=\left[\begin{array}{l}
\mathrm{E}_{\mathrm{z}} \\
\mathrm{D}_{\mathrm{z}}
\end{array}\right]
$$

This yields:

$$
\begin{aligned}
& a_{1}=\frac{D_{y}^{2} E_{z}-E_{y}^{2} D_{z}}{E_{y} D_{y}^{2}-D_{y} E_{y}^{2}} \\
& a_{2}=\frac{-D_{y} E_{z}-E_{y} D_{z}}{E_{y} D_{y}^{2}-D_{y} E_{y}^{2}}
\end{aligned}
$$

Now, we have determined the trial polynomial based on a trial position of point (D). We can trace this polynomial back in the direction of the base to locate the points (C) and (B), respectively as follows. Let (D) be the centre point of the circle whose radius is $\mathrm{L}_{3}$. The equation of this circle is

$$
\left(\mathrm{y}-\mathrm{D}_{\mathrm{y}}\right)^{2}+\left(\mathrm{z}-\mathrm{D}_{\mathrm{z}}\right)^{2}=\mathrm{L}_{3}^{2}
$$

As shown in Fig. 3, Eq. (8) intersects Eq. 1 at two points with point (D) is in the middle. The first point lies farthest from the base in the direction of the end effector (which must be ignored, since, it is in the undesired direction). The second point lies closer to the base which is selected to be the trial point $\mathrm{C}$ with coordinate

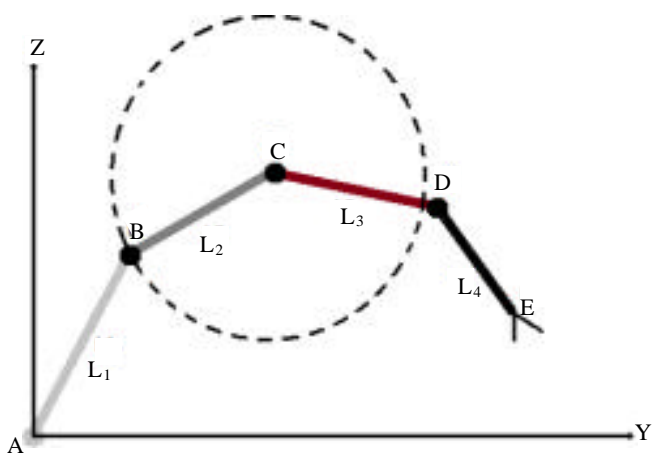

Fig. 4: Locating point $B$

set $\left(0, \mathrm{C}_{\mathrm{y}}, \mathrm{C}_{\mathrm{z}}\right)$. The points of intersection are located by solving Eq. 1 and 8 simultaneously resulting in a fourth degree polynomial of the form:

$$
\mathrm{b}_{0}+\mathrm{b}_{1} \mathrm{y}+\mathrm{b}_{2} \mathrm{y}^{2}+\mathrm{b}_{3} \mathrm{y}^{3}+\mathrm{b}_{4} \mathrm{y}^{4}=0
$$

Equation 9 has two real roots (which we are looking for) and another two imaginary roots with no significance to this problem. After we located the trial point $(\mathrm{C})$, we repeat the process to determine the location of point $(\mathrm{B})$ by Considering $(\mathrm{C})$ as the centre of the circle whose radius is $L_{2}$ (Fig. 4). The equation of this circle is:

$$
\left(\mathrm{y}-\mathrm{C}_{\mathrm{y}}\right)^{2}+\left(\mathrm{z}-\mathrm{C}_{\mathrm{z}}\right)^{2}=\mathrm{L}_{2}^{2}
$$

Equation 10 intersects Eq. 1 at two points one of them (the closest to the base) is the trial point $\mathrm{B}$ with coordinates $\left(0, B_{y}, B_{z}\right)$. Until now, all the points we found are only trial ones. For these trial points to be the true points, one condition have to be met, that is the distance between the trial point $B=\left(0, B_{y}, B_{z}\right)^{T}$ and the base point $\mathrm{A}=(0,0,0)^{\mathrm{T}}$ should equal exactly the length of the first Link (L1). If this was the case then the trial curve has successfully fulfilled the two constraints mentioned earlier. If not, the procedure must be repeated by selecting another trial point $\mathrm{D}$.

Testing the method: Two paths were chosen for the end effector to follow and the proposed method was used to determine the robotic arm inverse kinematics. The first path chosen is the one shown in Fig. 5 which is an ellipse in the $\mathrm{YZ}$ plane.

The path is traced beginning from the start point shown and moving in the anticlockwise direction then back to the starting point again. Figure 6 is the position profile which shows the variation of each joint angle of the robot as the robotic arm end effector traces the 

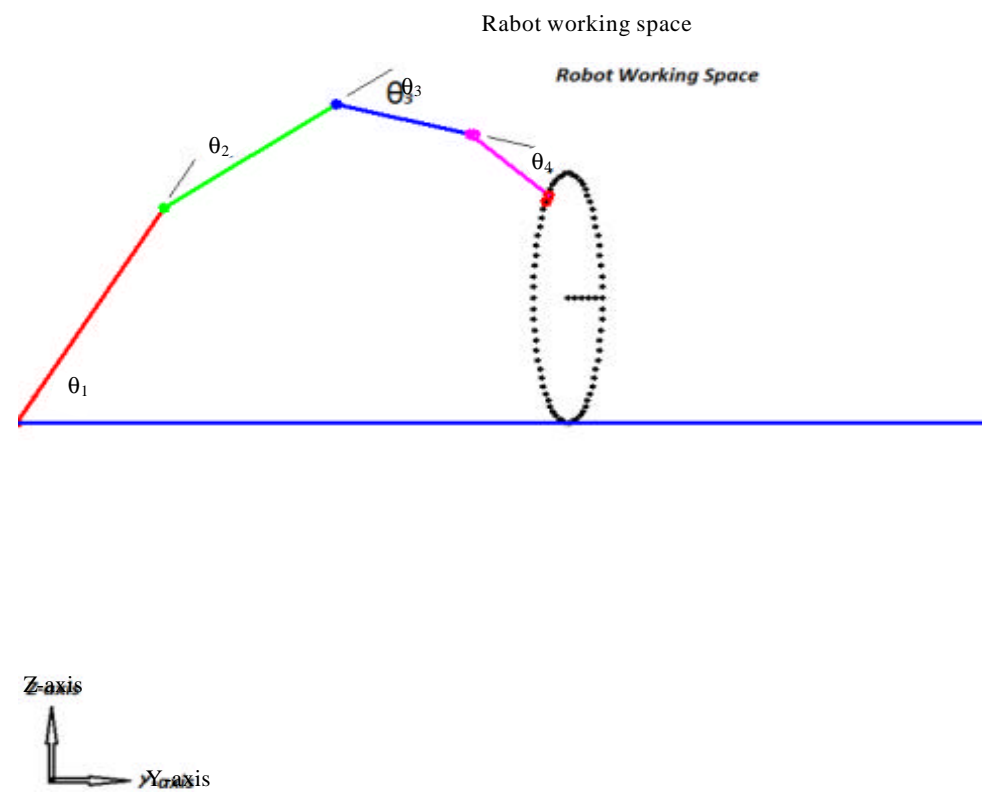

Fig. 5: YZ elliptic path

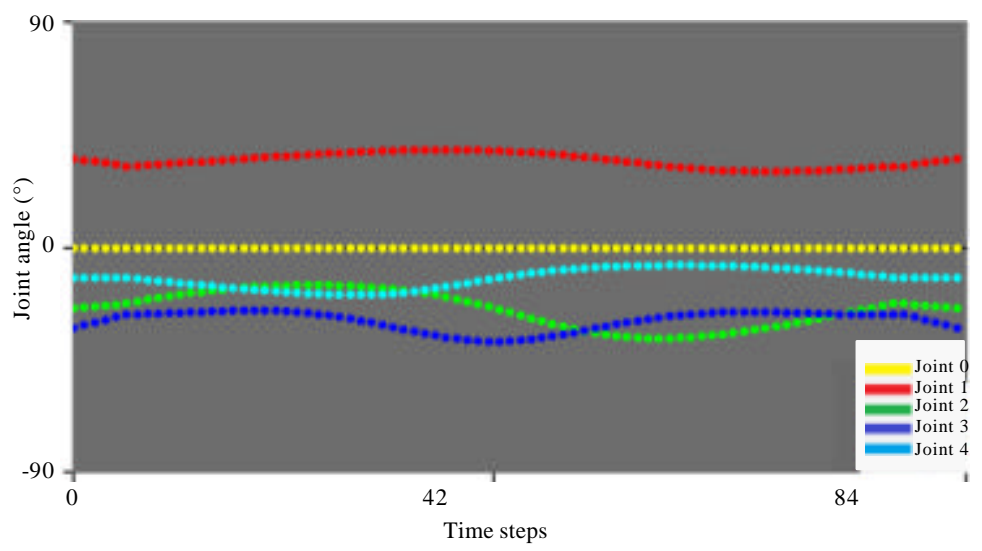

Fig. 6: The position profile corresponds to the path in Fig. 4

ellipse. Since, all the points of the path are in the YZ plane, the base angle is zero at every time step in the profile.

The method provides a smooth transition between any two consecutive configurations which is a very important requirement for a successful kinematic behavior of a robotic arm, since, it is directly related to the amount of torque required by the actuators at all the joints, the smoother variation in the angle the lower torque needed do drive the robotic arm along the path.

The second path chosen is shown in Fig. 7. It is an ellipse drawn in the XY plane. The path begins with the starting point and it is traced in the anticlockwise direction and ends with the same starting point. The position profile corresponds to this path is shown in

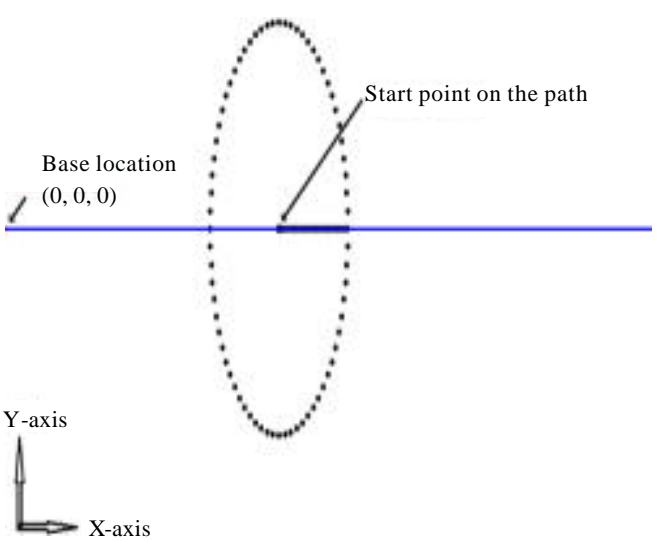

Fig. 7: XY elliptic path 


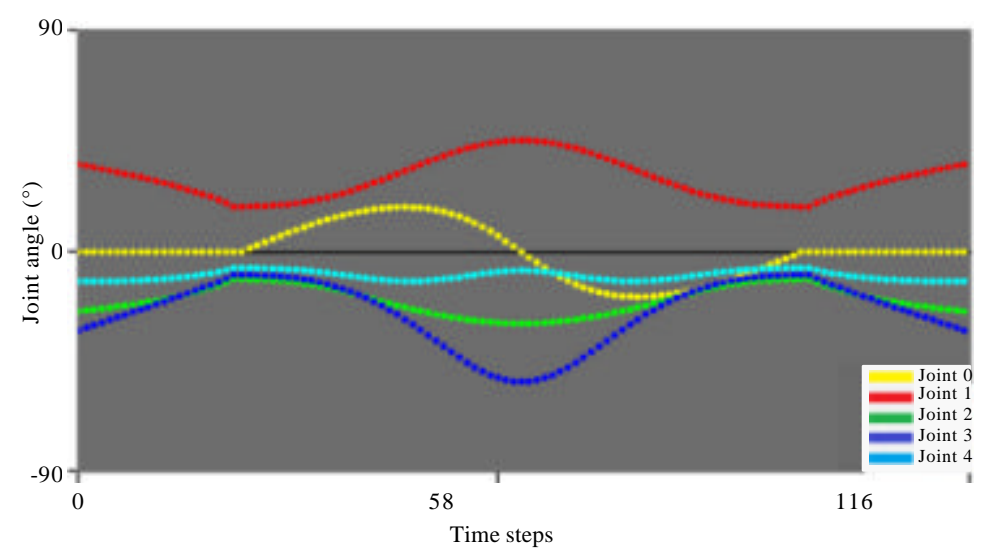

Fig. 8: Position profile corresponding to the path in Fig. 7

Fig. 8. As seen from the position profile, the method also provided smooth variation in all joint angles which means that the method proposed is very useful in solving the inverse kinematics problem for almost any path in the 3D working space of the robotic arm. This time the base angle varies during the motion.

\section{RESULTS AND DISCUSSION}

So far, we have demonstrated how a second order polynomial (which is an equation of a parabola) can be used to solve the inverse kinematics of a robotic arm with redundant links which is classified as a very hard-to-solve problem. According to the method, it is only required to evaluate the three parameters describing the polynomial, namely, $a_{0}-a_{2}$. However, using the second order polynomial has one drawback; the polynomial is not defined at the origin which makes all the points along the $\mathrm{Z}$-axis unreachable by the robotic arm. In fact, even the points near the Z-axis can be hard to reach considering the fact that at such close points, the second order, polynomial becomes very sharp in shape which results in some joint angles to be either too small or too large. In both cases, these extreme values of angles may become far beyond the allowable range of each joint angle for most open chain serial robotic arms.

The limitations of using a second order polynomial can be avoided by using another type of curves that overcome these problems, like the circle described by Eq. 11:

$$
(\mathrm{y}-\mathrm{H})^{2}+(\mathrm{z}-\mathrm{K})^{2}=\mathrm{R}_{2}{ }_{2}
$$

To apply the method using Eq. 11, it is only required to locate its centre at $(0, H, K)$ and the radius $R$. These values are also required to fulfill the constraints given in

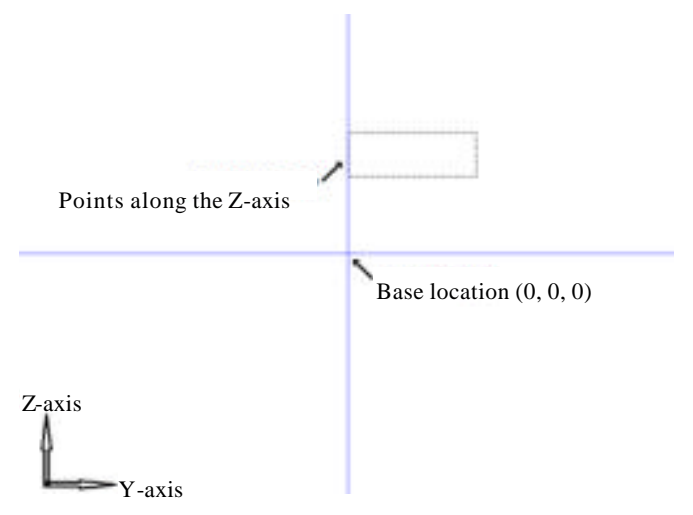

Fig. 9: Rectangular path

our earlier discussion for the case of the second order polynomial. Using the circular configuration enables the robotic arm to reach all the points within the working space except the base point of the arm which is already a forbidden point to reach or the robotic arm would hit itself.

To test the performance of the circular configuration, the path shown in Fig. 9 is assigned to a 5-DOF robotic arm which has the following characteristics:

- The path includes points that lie on the $\mathrm{z}$-axis

- The path selected is very close to the origin

A second order polynomial configuration will certainly fail to follow such a path. Figure 10 shows the configuration of the robotic arm reaching a point on the path. The resulting position profile after tracing all the points on the path by the end effector is shown in Fig. 11.

The position profile clearly shows the very smooth variation of all the five joints as the end effector traces the 


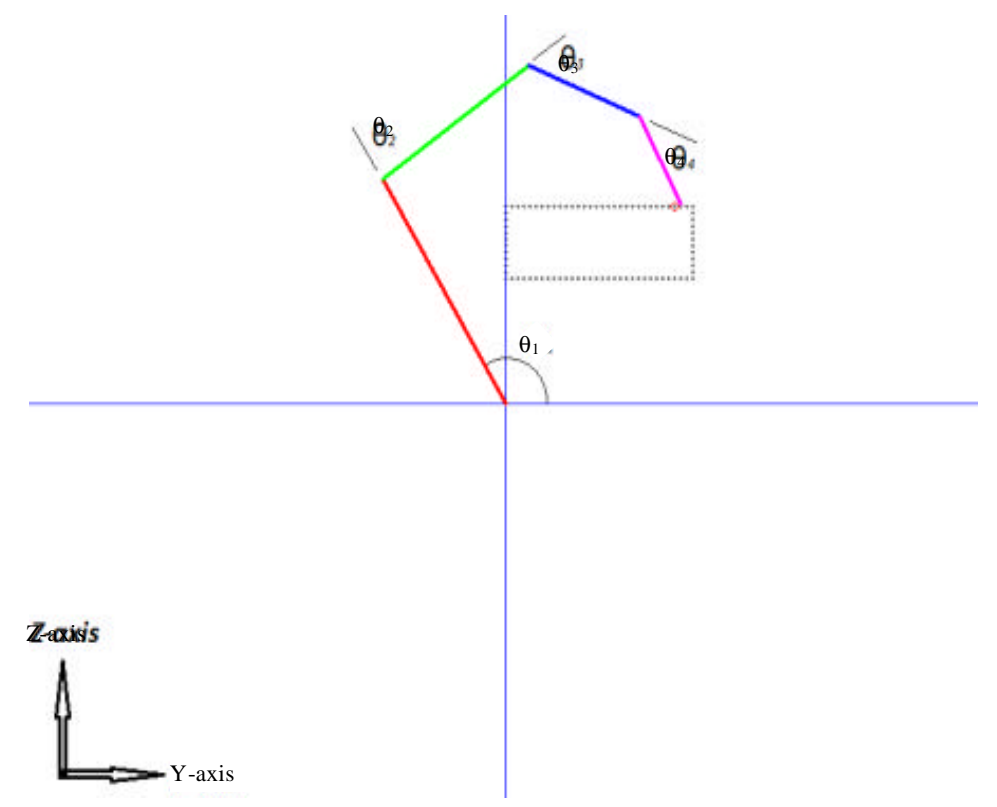

Fig. 10: The robotic arm following the rectangular path based on the circular configuration method

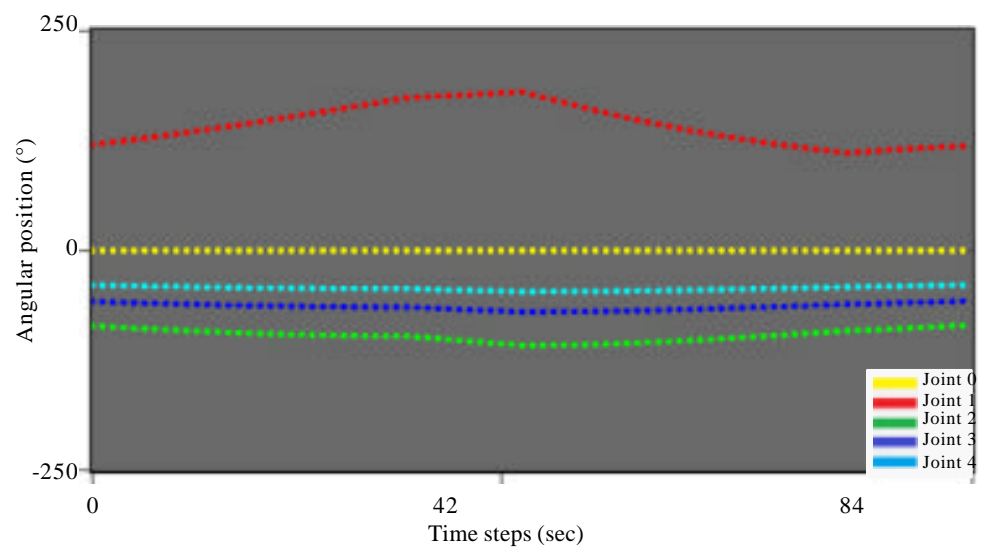

Fig. 11: The position profile for the robotic arm following the rectangular path based on the circular configuration method

path. This smooth transition from point to point has an advantage on the overall motion of the robotic arm in that it reduces the power consumption to drive the arm as a result of the reduction in the torque applied at each joint during motion. Also, the smooth variation in joint angles makes the robotic arm more stable during its motion which increases the accuracy of path following performance.

As a comparison, the 5-DOF robotic arm was given the same path shown in Fig. 5 but this time the circular configuration was used. Figure 12 shows the arm as it follows the given path. The figure shows the similarity of the arm configuration between the two cases (2nd order polynomial configuration and the circular configuration).

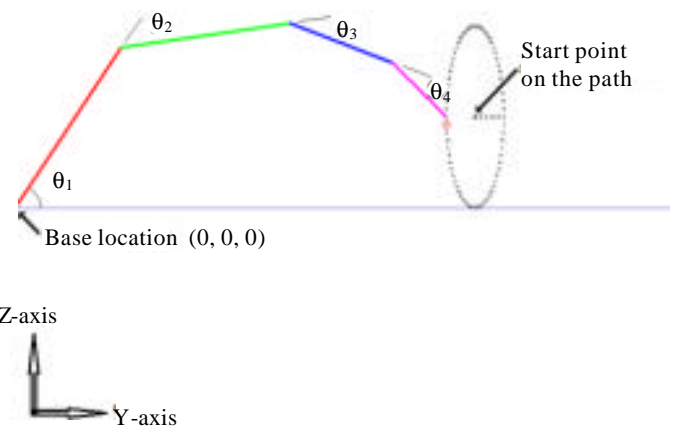

Fig. 12: The robotic arm following the path of Fig. 5 based on the circular configuration method 


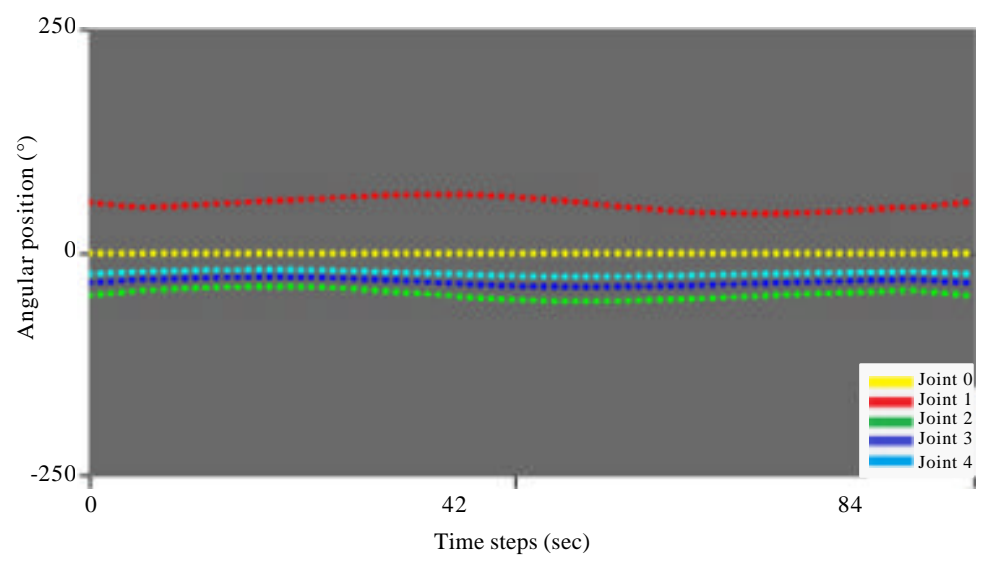

Fig. 13: The position profile for the robotic arm following the path in Fig. 5 based on the circular configuration method

The corresponding position profile is shown in Fig. 13. Despite taking a similar behavior upon following the path, the resulting position profile for the case of the circular configuration showed a much smoother variation in all the joints during the motion. This means that the circular configuration technique is a much better choice over the second order polynomial to drive the robotic arm. Since, the motion is only in the YZ-plane, the base has no rotation and its zero at all the time steps in the two profiles.

\section{CONCLUSION}

In this study, a new way of thinking was used to solve the inverse kinematics problem for open chain robotic arms. The inverse kinematics of this type of robots becomes very complicated and even impossible to solve as the degree of freedom increases. The method is based on selecting a curve in space and forcing the joints to align on it. The selection of the curve is dependent upon the available working space and on the design of the robotic arm itself. The method proposed overcomes the difficulties usually encountered in the case of increasing the degree of freedom of the robotic arm. In fact the method gave a very good performance in the process of positioning the end effector for, theoretically, any number of degrees of freedom the robotic arm may have. For demonstration purposes, however, the method was tested on a 5 -DOF robotic arm.

\section{ACKNOWLEDGEMENT}

I would like to express my appreciation for Dr. Hasan M. Alwan for his kind support in preparing this paper as a supervisor and a scientific guidance. Also, I have to mention the role of Dr. Qasim A. Ateia who helped me in programming the algorithms I made to accomplish the final goals of the research.

\section{REFERENCES}

Basu, R. and S. Padage, 2017. Development of 5 DOF Robot Arm-Gripper for sorting and investigating RTM concepts. Mater. Today Proc., 4: $1634-1643$.

Crenganis, M., R.E. Breaz, S.G. Racz and O. Bologa, 2014. Inverse Kinematics for a 7 DOF Robotic Arm using the Redundancy Circle and ANFIS Models. In: Applied Mechanics and Materials, Pelz, P.F. and P. Groche (Eds.). Trans Tech Publications, Switzerland, pp: 823-828.

Csiszar, A., J. Eilers and A. Verl, 2017. On solving the inverse kinematics problem using neural networks. Proceedings of the 2017 24th International Conference on Mechatronics and Machine Vision in Practice (M2VIP), November 21-23, 2017, IEEE, Auckland, New Zealand, ISBN:978-1-5090-6547-9, pp: $1-6$.

Das, L. and S.S. Mahapatra, 2015. Prediction of inverse kinematics of a 5-DOF pioneer robotic arm having 6-DOF end-effector using ANFIS. Intl. J. Comput. Vision Rob., 5: 365-384.

Drexler, D.A., 2016. Solution of the closed-loop inverse Kinematics algorithm using the Crank-Nicolson method. Proceedings of the 2016 IEEE 14th International Symposium on Applied Machine Intelligence and Informatics (SAMI), January 21-23, 2016, IEEE, Herlany, Slovakia, ISBN:978-1-4673-87408, pp: 351-356.

Duka, A.V., 2014. Neural network based inverse kinematics solution for trajectory tracking of a robotic arm. Procedia Technol., 12: 20-27. 
Ligutan, D.D., L.J.S. Cruz, M.C.D.P. Del Rosario, J.N.S. Kudhal and A.C. Abad et al., 2017. Design and implementation of a fuzzy logic-based joint controller on a 6-DOF robot arm with machine vision feedback. Proceedings of the 2017 International Conference on Computing, July 18-20, 2017, IEEE, London, UK., ISBN:978-1-5090-5444-2, pp: 249-257.

Patil, A., M. Kulkarni and A. Aswale, 2017. Analysis of the inverse kinematics for $5 \mathrm{DOF}$ robot arm using $\mathrm{DH}$ parameters. Proceedings of the 2017 IEEE International Conference on Real-time Computing and Robotics (RCAR), July 14-18, 2017, IEEE, Okinawa,Japan,ISBN:978-1-5386-2036-6,pp: 688-693.

Proceedings of the 2017 Congress on Chinese Automation (CAC), 2017. Inverse kinematics solution for six-DOF serial robots based on BP neural network. Proceedings of the 2017 Congress on Chinese Automation (CAC), October 20-22, 2017, IEEE, Jinan, China, ISBN:978-1-53863525-4, pp: 1154-1157.
Raj, D.R., I.J. Raglend and M.D. Anand, 2015. Inverse kinematics solution of a five joint robot using feed forward and Elman network. Proceedings of the 2015 International Conference on Circuits, Power and Computing Technologies [ICCPCT- 2015], March 1920, 2015, IEEE, Nagercoil, India, ISBN:978-1 -47997075-9, pp: 1-5.

Reiter, A., A. Muller and H. Gattringer, 2018. On higher order inverse kinematics methods in timeoptimal trajectory planning for kinematically redundant manipulators. IEEE. Trans. Ind. Inf., 14: 1681-1690.

Wang, Y. and L. Wang, 2017. Applicability analysis of generalized inverse kinematics algorithms with respect to manipulator geometric uncertainties. Proceedings of the 2017 IEEE/RSJ International Conference on Intelligent Robots and Systems (IROS), September 24-28, 2017, IEEE, Vancouver, Canada, ISBN:978-1-5386-2683-2, pp: 2813-2820. 